\title{
THE MINIMUM TOTAL HEATING LANDER
}

\author{
DANG THI MAI \\ Faculty of Basic Sciences - University of Transport and \\ Communications; Maidt.utc@gmail.com
}

\begin{abstract}
The article will research a lander flying into the atmosphere with flow velocity constraint, i.e. the total load by means of minimizing the total thermal energy at the end of the landing process. The lander's distance at the last moment depends on the variables selected from the total thermal energy minima. To deal with the problem, the Pontryagin maximum principle and scheme Dubovitskij Milutin will be applied. Boundary value problems are solved by the introduction and continuation of the perturbation parameters and solutions for the selected parameter. The results of simulations perform on Matlab.
\end{abstract}

Keywords. Maximum principle, control, the overload, total heat, minimum.

\section{INTRODUCTION}

Research is on the problem of choosing an angle to launch the flying object which is reducing velocity in atmospheric conditions under which the minimizing of total heat flow with the load limits of aircraft equipment is taken into account. The total heat output of the device is the integral form of the following:

$$
Q=\int_{0}^{T} C V^{3} \rho^{\frac{1}{2}} d t
$$

Required to detemine a control $C_{y}(t)$, which minimizes $Q(T)(1)$ under the following restrictions:

$$
\begin{gathered}
n_{\sigma}=\sqrt{C_{(x)}^{2}+C_{y}^{2}} \dot{q} \frac{S}{G} \leq N, \quad q=\frac{\rho V^{2}}{2}, \quad G=m g, \\
C_{y}^{\min } \leq C_{y} \leq C_{y}^{\max }, \quad C_{x}=C_{x 0}+k C_{y}^{2}, \\
\rho=\rho_{0} e^{-\beta H}, \quad g=g_{0} \frac{R^{2}}{(R+H)^{2}}, \quad \dot{V}-C_{x} q \frac{S}{m}-g \sin \theta \\
\dot{\theta}=C_{y} q \frac{S}{m V}+\left(\frac{V}{R+H}-\frac{g}{V}\right) \cos \theta, \dot{H}=V \sin \theta \\
\dot{L}=\frac{R V \cos \theta}{R+H}
\end{gathered}
$$

where $n_{\sigma^{-}}$full overload, $q$ - speed pressure, $\rho$ - atmospheric density, $V$ - velocity of the vehicle, $\theta$ path angle, $H$ - height, $L$ - the remote, G- the weight of the machine, $m--$ mass, $g_{0}$ - acceleration due to gravity on the surface of the planet, $R$ - the radius of the planet, $C_{x^{-}}$the drag coefficient, $C_{y^{-}}$ lift coefficient, $S$ - characteristic area apparatus, $C_{x 0}, k, \rho_{0}, \beta, C_{y}^{\min }, C_{y}^{\max }, N$ - constants.

(C) 2015 Vietnam Academy of Science \& Technology 
For the system (1) - (5) the initial conditions:

$$
V(0)=V_{0}, \quad \theta(0)=\theta_{0}, \quad H(0)=H_{0}, \quad L(0)=L_{0}, \quad Q(0)=0
$$

and conditions and limitations:

$$
L(T)=a, \quad V(T)=V_{1}, \quad \theta(T)=\theta_{1}, \quad H(T)=H_{1}, \quad T-\text { not fixed } .
$$

where $a$ - parameter.

\section{APPLICATION OF MAXIMUM PRINCIPLE IN THE REGULAR CASE}

Let lander come from the initial state (7) in a washed-position (8) in an optimal way in the sense of minimizing the total amount of heat under the assumption of optimal trajectory regularity condition $[1,2]$. In the above problem, the regularity condition is equivalent to

$$
\frac{\partial n_{\sigma}}{\partial C_{y}} \neq 0, n_{\sigma}=N
$$

In this case, the maximum principle is as follows:

$$
\begin{gathered}
\Pi=P_{\theta} \dot{\theta}+P_{H} \dot{H}+P_{V} \dot{V}+P_{L} \dot{L}+P_{Q} \dot{Q}, \quad L_{1}=\Pi=-\lambda(t)\left(n_{\sigma}-N\right) \\
P_{\theta}^{\bullet}=-\frac{\partial \Pi}{\partial \theta}, \quad P_{V}^{\bullet}=-\frac{\partial \Pi}{\partial V}, \quad P_{H}^{\bullet}=-\frac{\partial \Pi}{\partial H}, \quad P_{L}^{\bullet}=-\frac{\partial \Pi}{\partial L}, \quad P_{Q}^{\bullet}=-\frac{\partial \Pi}{\partial Q} .
\end{gathered}
$$

Here $\lambda(t)$ - the Lagrange multiplier, which is determined from the condition of Bliss $[1,2]$.

$$
\frac{\partial \Pi}{\partial C_{y}}-\lambda(t) \frac{\partial n_{\sigma}}{\partial C_{y}}=0
$$

$\Pi$-Pontryagin function, $L_{1}$ - Lagrange function.

$P_{\theta}, P_{V}, P_{H}, P_{L}, P_{Q}$ - corresponding conjugate variables. For inequality constraints (2) satisfies the complementary slackness.

$$
\lambda(t)\left(n_{\sigma}-N\right)=0
$$

Since the system (1) - (6) is autonomous and there is no descent of restrictions, the Pontryagin function (10) is identically zero, i.e.

$$
\Pi(P, x, u) \equiv 0, \quad u=C_{y}, \quad x=\left(\theta, V, H_{y}, L\right), \quad P=\left(P_{\theta}, P_{V}, P_{H}, P_{L}, P_{q}\right)
$$

Conjugate variable $P_{Q}(t)$ normalized by the condition

$$
P_{Q}(t) \equiv-1
$$

The initial conditions for the system (11) are unknown parameters of the problem. Condition $P_{Q}(t) \equiv-1$ and $\Pi(P, x, u) \equiv 0$ is essentially determined by three free parameters

$$
P_{\theta}(0)=C_{1}, \quad P_{V}(0)=C_{2}, \quad P_{L}(0)=C_{3}
$$

since $P_{H}(0)$ is determined from the condition $\Pi(P, x, u) \equiv 0$. 
In this case, the number of controlled functions at the end of the trajectory (8) coincides with the number of free parameters of the problem (1) - (8), (10), (11), because the time $\mathrm{T}$ is not fixed and is a free parameter.

According to the principle of maximum control program chosen from the condition:

$$
\Pi \rightarrow \max _{C_{y}} \text { while } Q(T) \rightarrow \min
$$

The part Pontryagin function (10) can be written down, which clearly depends on the control $C_{y}(t)$.

$$
\Pi_{0}=P_{\theta} \frac{C_{y} \rho V S}{2 m}-P_{V} \frac{C_{x} \rho V^{2} S}{2 m}
$$

$C_{y}(t)$ can take control of not only limit values (3), but also an intermediate, which is determined from the condition

$$
\frac{\partial \Pi_{0}}{\partial C_{y}}=0, \quad C_{y}^{*}=\frac{P_{\theta}}{2 k P_{V} V}, \quad C_{y}^{\min }<C_{y}^{*}<C_{y}^{\max }
$$

Three values of the function $\Pi_{0}$ are calculated in (18)

$$
\Pi_{1}=\Pi_{0}\left(C_{y}^{\min }\right), \quad \Pi_{2}=\Pi_{0}\left(C_{y}^{\max }\right), \quad \Pi_{3}=\Pi_{0}\left(C_{y}^{*}\right)
$$

and

$$
\Pi_{0}^{\max }=\max \left\{\Pi_{1}, \Pi_{2}, \Pi_{3}\right\}
$$

Equation (20) determines the nature of the optimal control problem of Pontryagin, i.e. provided that $n_{\sigma} \leq N$. Solution to the problem is greatly simplified if the right end of the trajectory is controlled by the condition

$$
H(T)=H_{1}
$$

In this case, the solution to (1) - (8) is determined by the boundary conditions

$$
\theta(T)=\theta_{1}, \quad V(T)=V_{1}, \quad L(T)=a
$$

and depends on three arbitrary constants $C_{1}, C_{2}$ and $C_{3}$.

Thus, the initial problem is reduced to a three-parameter problem (1) - (8), (16), (11), (22), and the optimal control $C_{y}(t)$ is determined at each point $\mathrm{t}$ of the maximum principle (22).

\section{RESTRICTION ON OVERLOAD}

The task difficulty of determining the geometry of optimal trajectory is the identification of points coming off the disabled $n_{\sigma}=N$.

Note that the total overload (2) has two components $n_{x}$ and $n_{y}$. The first is called a longitudinal overload, and the second - normal.

$$
n_{y}=\frac{\rho V^{2} S}{2 m g_{0}} C_{y}, \quad n_{x}=\frac{\rho V^{2} S}{2 m g_{0}} C_{x}, \quad n_{\sigma}=\sqrt{n_{x}^{2}+n_{y}^{2}} .
$$

Instead of limiting (2), a new restriction is introduced

$$
\left|n_{y}\right|+n_{x} \leq N_{1}, \quad\left|n_{y}\right|+n_{x}-N_{1}=\varphi(x, u) \leq 0
$$


With an appropriate choice $N_{1}$ of the inequality (24) is known to be satisfied constraint (2). This fact follows from

$$
N_{1} \geq\left[\left|n_{y}\right|+\left|n_{x}\right|\right] \geq \sqrt{n_{x}^{2}+n_{y}^{2}}
$$

equal sign occurs when $C_{y}=0$.

Now, it is to compute the derivative of $\varphi(x, u)(24)$ following $C_{y}$

$$
\frac{\partial \varphi}{\partial C_{y}}=\frac{\rho V^{2} S}{2 m g_{0}}\left[\operatorname{sign} C_{y}+2 k C_{y}\right]
$$

In this case, the Lagrange multiplier $\lambda(t)$ for limiting $\varphi(x, u) \leq 0(24)$ is determined by the formula

$$
\lambda(t)=\frac{2\left(\frac{P_{\theta}}{2}-k P_{V} C_{y} V\right) g_{0}}{V\left[\operatorname{sign} C_{y}+2 k C_{y}\right]}
$$

\section{NECESSARY OPTIMALITY CONDITIONS IN THE IRREGULAR CASE}

Now consider the case when the optimal trajectory contains an interval, when $n_{\sigma}=N$ and in this interval at some point $\frac{\partial n_{\sigma}}{\partial C_{y}}=0$.

The set of points defined by the equations

$$
\frac{\partial n_{\sigma}}{\partial C_{y}}=0, \quad n_{\sigma}=N
$$

following [1], it is to call irregular points. For the problem $\frac{\partial n_{\sigma}}{\partial C_{y}}=0$ at $C_{y}=0$. For the given problem the results of of A. I. Dubovitskij and A. A. Miliutin [1,2] can be used. According to Refs. $[1,2]$ in the presence of irregular points, conjugate system of equations is

$$
\begin{aligned}
\dot{P}_{\theta} & =-\frac{\partial}{\partial \theta} \\
\dot{P}_{H} & =-\frac{\partial}{\partial H}+\lambda(t) \frac{\partial n_{\sigma}}{\partial H}+\frac{d \mu}{d t} \frac{\partial n_{\sigma}}{\partial H} \\
\dot{P}_{V} & =-\frac{\partial}{\partial V}+\lambda(t) \frac{\partial n_{\sigma}}{\partial V}+\frac{d \mu}{d t} \frac{\partial n_{\sigma}}{\partial V} \\
\dot{P}_{L} & =0 \\
\dot{P}_{Q} & =0 .
\end{aligned}
$$

Here - $\lambda(t)$ Lagrange multiplier - a $\frac{d \mu}{d t}$ generalized function. For these objects, complementary slackness condition is made

$$
\lambda(t)\left(n_{\sigma}-N\right)=0, C_{y} \frac{d \mu}{d t}=0 .
$$

From (29) it follows that in the irregular point (28) and the conjugate variables will experience racing on the values of $\mu \frac{\partial n_{\sigma}}{\partial H}$ and $\mu \frac{\partial n_{\sigma}}{\partial V}$ when $\mu>0$. This is the essential difference between the case of irregular regular, where the conjugate variables are continuous functions for mixed class constraints $[1,2]$.

Besides the conditions (28) - (30) the optimal trajectory should be the conditions of integrability of the Lagrange multipliers and the normalization condition (non-triviality condition of the maximum principle). 


\section{REGULARIZATION DEGENERATE OF THE MAXIMUM PRINCIPLE}

One of the possible ways of constructing a nondegenerate optimal trajectory is to change the structure of restriction (28). Limitation (29) has been used previously for sustainable iterative search for the optimal trajectory for small $C_{y}(t)$. This Lagrange multiplier is calculated by the formula (27). Changes in the structure of mixed constraints (23) do not impose additional requirements on the function $P_{\theta}(t)$ in an irregular point $\left(P_{\theta}\left(t_{*}\right)=0\right)$. However, to continue the path through the point $t_{*}$ is necessary to satisfy the condition $q^{\bullet}\left(t_{*}\right)=0$. As a result, there are three conditions on an irregular optimal trajectory

$$
\dot{q}\left(t_{*}\right)=0, \quad P_{V}(T)=0, \quad P_{\theta}(T)=0 .
$$

that can be performed by selecting the jumps conjugate variables of the form (16) and arbitrary constants $P_{V}(0), P_{\theta}(0)$.

With this approach, a non-degenerate maximum principle throughout the optimal trajectory can be gotten.

The presence of several irregular points also leads to degeneration of the maximum principle, however, complicates the search for the optimal trajectory.

Let us now consider another approach to the construction of a non-degenerate maximum principle. For this purpose, the construction of Pontryagin function (10) value $P_{\theta} \frac{C_{y} \rho V S}{2 m}$

considers a small parameter at sufficiently small $C_{y}(t)$. Then the expression for the Lagrange multiplier $\lambda(t)$ takes the form:

$$
\lambda(t)=-\frac{2 k P_{V}}{1+2 k C_{x}} g_{0} \sqrt{C_{x}^{2}+C_{y}^{2}}
$$

In this case, the integrability conditions $\lambda(t)$ are performed automatically.

The result is a non-degenerate maximum principle with irregular points. In addition, the expression (32) allows for a steady iterative search for the optimal trajectory for small.

Another way of regularization of the degenerate maximum principle is mentioned. Suppose that the optimal trajectory condition $n_{\sigma}=N(2)$, then it yields

$$
\frac{1}{2} \ln \left(C_{x}^{2}+C_{y}^{2}\right)+\ln \frac{\rho V^{2} S}{2 m g_{0}}=\ln N
$$

Now consider separately the members of (33), which are associated with the management of

$$
\begin{aligned}
\frac{1}{2} \ln \left(C_{x}^{2}+C_{y}^{2}\right) & =\ln \left[\left(C_{x}+C_{y}\right)^{2}-2 C_{y} C_{x}\right] \\
& =\frac{1}{2} \ln \left(C_{x}+C_{y}\right)^{2}\left[1-\frac{2 C_{y} C_{x}}{\left(C_{y}+C_{x}\right)^{2}}\right] \\
& =\ln \left(C_{x}+C_{y}\right)+\frac{1}{2} \ln \left[1-\frac{2 C_{y} C_{x}}{\left(C_{y}+C_{x}\right)^{2}}\right]
\end{aligned}
$$

These expressions do not have singularities at $C_{y}=0$. This means that in this case a Lagrange multiplier for the constraint (33) will be finite. Thus, the irregular point does not impose any restrictions on the conjugate variables $P_{\theta}(t)$. The result is a non-degenerate maximum principle. 


\section{EXAMPLE AND NUMERICAL RESULT}

For more details of the problem, it is to solve the problem of finding the minimum maximum total heating of space shuttle $[3,5]$, constants and the boundary conditions are:

$$
\begin{aligned}
& C_{y}^{\text {min }}=-0.5 ; C_{y}^{\text {max }}=0.6 ; \frac{S}{m}=50000 ;\left[\mathrm{km}^{2} \mathrm{~kg}^{-1}\right] ; \rho_{0}=2.3769 \times 10^{-3} \\
& R=6371.2[\mathrm{~km}] ; C_{x 0}=0.88 ; k=0.5 ; g_{0}=9.8 \times 10^{-3}\left[\mathrm{kms}^{-2}\right] \\
& C=20 ; N=4 ; \beta=0.145 ; \theta(0)=-1.25[\mathrm{deg}] ; V(0)=0.35[\mathrm{~km}] ; \\
& H(0)=100[\mathrm{~km}] ; L(0)=0[\mathrm{~km}] ; Q(0)=0 ;
\end{aligned}
$$

The result received the following number:

Figure 1 illustrates the shuttle's altitude over time, it is seen that the height $\mathrm{H}$ decreases rapidly from100 $\mathrm{km}$ down to $40 \mathrm{~km}$ over a period [0, $200 \mathrm{~s}]$. Figure 2 shows the velocity of the shuttle which also drops significantly during this period..

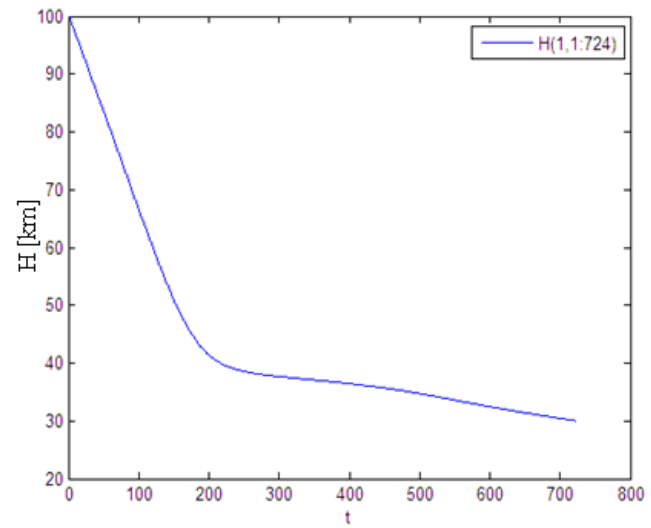

Figure 1: Height $\mathrm{H}[\mathrm{km}]$

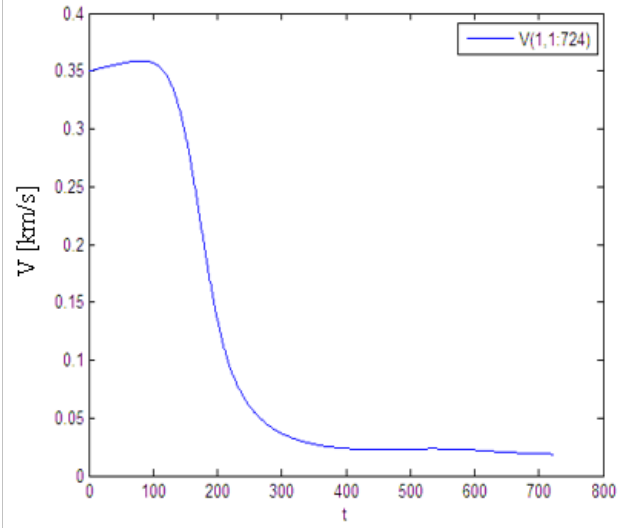

Figure 2: Velocity V[km/s]

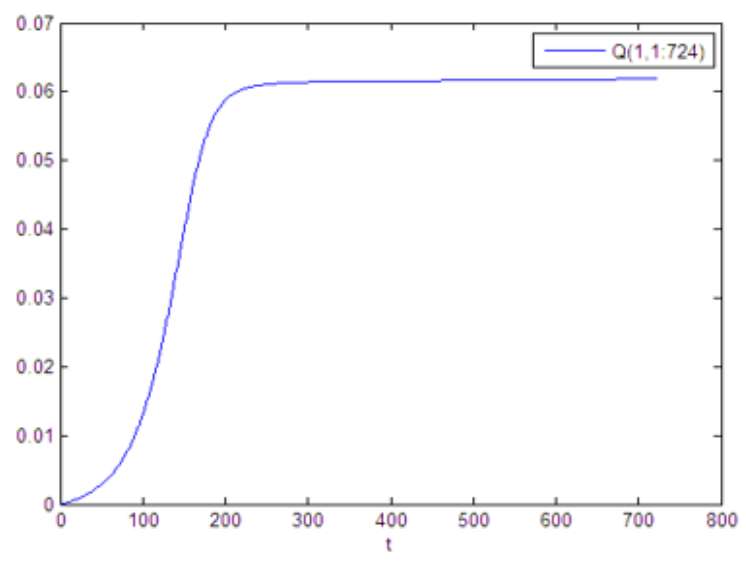

Figure 3: Total heat $Q(t)$

In Figure 3 , in the interval $[0,200 \mathrm{~s}]$ the total amount of surface temperature increases and stabilizes the ship during the period close to landing [200-720s]. According to simulations, the heat at the surface of the vessel can be considered to have been minimized during landing. 


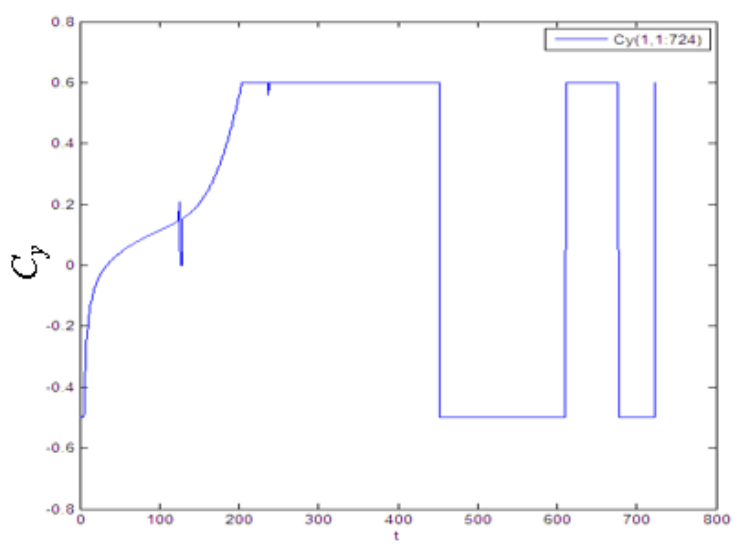

Figure 4: Lift coefficient $C_{y}(t)$

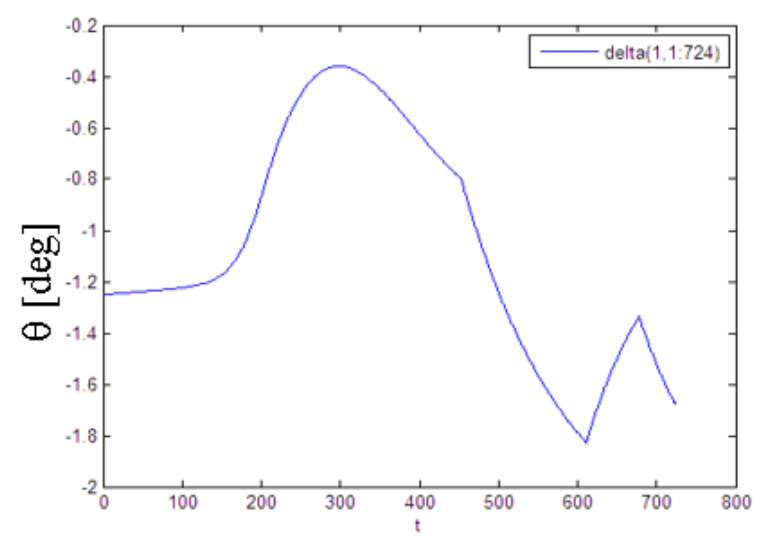

Figure 6: Path angle $\theta$

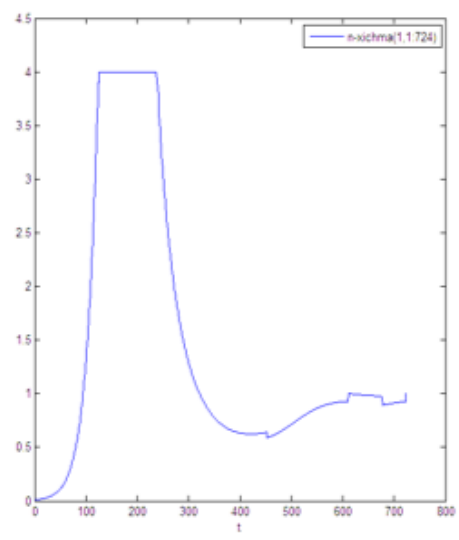

Figure 5: Full overload $n_{\sigma}$

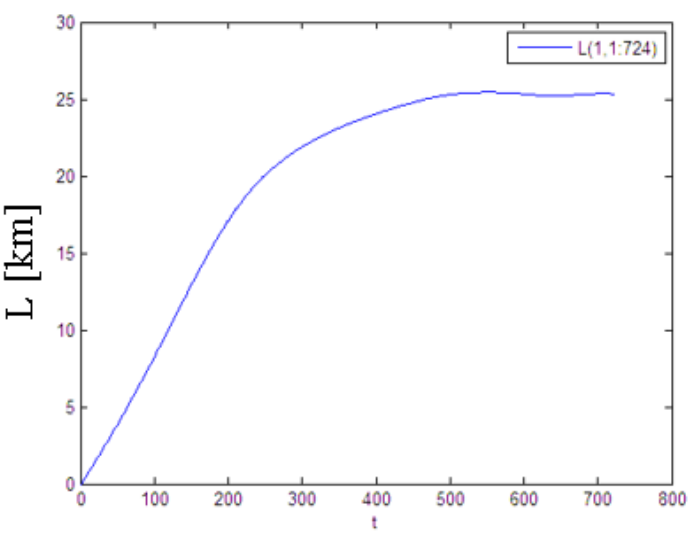

Figure 7: Remote $L[\mathrm{~km}]$

Figure 4 illustrates the state of the control variables changing over time and Figure 7 shows the remote of the space shuttle.

\section{CONCLUSIONS}

This paper solves the problem of minimizing the total heating (1) with the constraints (2) - (8) by using the Pontryagin maximum principle and Dubovistkij Miliutin system and the result is illustrated by numerical solution of software Matlab. Three parameter boundary value (22), the problem is solved for a fixed value $a$. Next, the desired value $a$ can be chosen from the minimum of the minimum value of the functional (1). The boundary value problem is solved by the continuation of solutions to the parameter [4].

\section{REFERENCES}

[1] Афанасьев А.П., Дикусар В.В., Милютин А.А., Чуканов С.В. Необходимое условие в принципе максимума. - М.: Наука, 1990. 
[2] Дикусар В.В., Милютин А.А. Количественные и качественные методы в принципе максимума - М.: Наука, 1989.

[3] O. Von Struk and R. Bulirsch. Direct and indirect methods for trajectory optimization. Annals of Operations Research 37(1992)357-373

[4] Дикусар В. В, Кошька М, Фигура А. Продолжение решений в прикладных задач оптимального управления. М., МФТИ. 2001.

[5] M. H Breitner and H. Josef Pesch. Reentry trajectory optimization under atmospheric uncertainty as a differential Game. Advances in dynamic games and applications, 1994.

Received on October 16 - 2014

Revised on December 20 - 2015 